\title{
POSITIONING DISASTER PREVENTION BASES IN PUBLIC FACILITIES USING VOLONOI DIAGRAM AND ANALYTIC NETWORK PROCESS
}

\author{
Kyouichi Himura and Takao Terano \\ Graduate School of Systems Management, University of Tsukuba, Tokyo \\ 3-29-1 Otsuka, Bunkyo-ku, Tokyo 112-0012 Japan \\ Tel: +81-3-942-6855, Fax: +81-3-942-6829, \\ Email: terano@gssm.otsuka.tsukuba.ac.jp.
}

\begin{abstract}
This paper proposes a method to solve the strategic positioning of disaster prevention functions prepared in public facilities. The method utilizes both the concepts of Volonoi diagram and Analytic Network Process. The proposed method is applied to the case of Mitaka city and reveals that the positioning recommended by the method is both useful and explainable.
\end{abstract}

\section{Introduction}

This paper discusses the issues in the strategic positioning of disaster prevention functions prepared in public facilities. The strategic positioning using public facilities intrinsically involves difficult multiobjective decision problems constraint by population distribution in a city, attributes of residents, hazard levels of regions, capacities and functions of disaster control centers, and the desires of both administrators and residents in a city.

To address the issues, this paper proposes a novel method to solve the problem using both the concept of Volonoi diagram [Iri 1994] and Analytic Hierarchy Process [Saaty 1996]. The most unique feature of the method is that it utilizes both of the information of pairwise comparison of a decision maker and the quantitatively measured data acquired from the survey study of the region.

\section{Outline of the Proposed Method}

The strategic positioning problems we consider in this paper are difficult in the sense that (1) there are few measurable data available to make the decisions, (2) there are conflicts among residents and/or regional administrators, and (3) The levels of disaster manegement are always varying from time to time. However, survey studies on Hanshin-Awaji Great Earthquake have revealed that behaviors of residents just after the earthquake can be explained by the concepts of Volonoi diagram, that is, people tend to move to geographically most nearest facilities just after the earthquake. These discussions support that when solving the positioning problems; we should use pairwise comparison based on the subjective judgements via AHP and/or ANP and that Volonoi diagram should be the one of the objective measures.

Based on above, we propose the following procedure to determine the positions of disaster prevention bases.

[Step 1] Determine the evaluation measures.

[Step 2] Determine the candidates of positioning patterns. Then draw the corresponding Volonoi diagrams.

[Step 3] Evaluate the positioning patterns based on the evaluation measures. That is, using pairwise comparison by a decision maker and/or based on the ratio of relative measures, develop comparison matrices.

[Step 4] Evaluate the evaluation measures based on the positioning patterns. That is, using pairwise 
comparison by a decision maker and/or based on the ratio of relative measures, develop comparison matrices.

[Step 5] Using the matrices in [Step 3 \& 4], develop a super matrix for ANP.

[Step 6] Applying ANP to the matrix in [Step 5], Get the evaluation results.

\section{Experiment and Discussion}

We have applied the proposed method to the case of Mitaka city to design the positioning the disaster prevention centers. Mitaka city has four major administration offices, which are the candidates of main disaster prevention bases and sixteen schools, which are capable of gathering residents proportional with the number of the students. Using the four and sixteen candidates, we develop two kinds of Volonoi diagram. Related information on the disaster management are associated to each of the regions of the diagrams.

The decision problem we formulate is that which is the best pattern of main disaster prevention bases selected from the one, two, three candidates among the four offices. The evaluation measures selected are rescue manager positioning, maximum distance to the bases, usefulness from the residence, efficiency from the administrators, the geographical deviation of the bases, and the number of residents corresponding bases. Please note that some of the measures are subjective and the others are quantitatively obtainable. However, using the proposed method, we have succeeded in determining desirable positioning patterns, which are seemingly counter intuitive, however, correct from intensive evaluation via other statistical data.

\section{Concluding Remarks}

This paper has addressed the issues of the strategic positioning problems on disaster prevention bases in public facilities of a city. We have proposed a procedure to determine the positions based on the concepts of ANP and Volonoi diagrams.

The proposed method is characterized by the mixed utilization of both subjective and quantitatively measurable data. The method has been evaluated by the case studies of Mitaka city in Tokyo area. Such a procedure will be effective to make decisions on uncertain political task domain problems. Future work includes (1) to apply the method to more complex situations and (2) to implement decision support tools based on the techniques.

\section{References}

[Iri 1993] Iri, M., Koshizuka, T.: Computational Geometry and Geographic Information Processing (2nd Ed.). Kyouritsu-Shuppan Pub. (in Japanese), 1993.

[Saaty 1996] Saaty, T. L.: The Analytic Network Process, RWS Publication, 1996. 